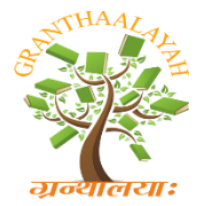

\author{
INTERNATIONAL JOURNAL OF RESEARCH - \\ GRANTHAALAYAH \\ A knowledge Repository
}

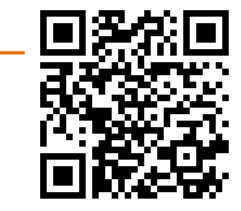

Science

\title{
A SURVEY ON FINGERPRINT PATTERN RECOGNITION
}

\author{
Swarnadip Dey ${ }^{1}$, Sajal Kumar Karmakar ${ }^{2}$, Surajit Goon ${ }^{3}$, Prianka Kundu ${ }^{* 4}$ \\ 1, 2,3,4 Department of Computer Application, Eminent College of Management \& Technology, \\ Barasat, Kolkata, West Bengal 700126, India
}

\begin{abstract}
In this advance technical time, we all need accuracy to any security system. Among all security system, biometric recognition process is very popular in that time. Not only security purpose, identification is the main cause of using biometric characteristic. A pin, password combination is not enough to secure all things because that's tracking is possible, but a person biometric characteristic is unique, so it is near to impossible to by-pass. In this paper, we discuss about the fingerprint types such as arch, loop, and whorl. We also discuss how the fingerprint will be recognized; however, where this technique is used in very large scale and what is the future scope of this technique, we discuss what improvement is needed in future.
\end{abstract}

Keywords: Biometric Recognition; Plain Whorl; Ulnar Loop; Plain Arch.

Cite This Article: Swarnadip Dey, Sajal Kumar Karmakar, Surajit Goon, and Prianka Kundu. (2019). "A SURVEY ON FINGERPRINT PATTERN RECOGNITION." International Journal of Research - Granthaalayah, 7(8), 496-506. 10.29121/granthaalayah.v7.i8.2019.704.

\section{Introduction}

Biometric characteristic is the best way to identify any individual person, however all biometric characteristics are not best option to identification because some biometric attributes are change by time. Mridula, Priyanka paper [1] gives some biometric attributes like voice, signature are changeable, however some are not that's his fingerprint, bite marks, lip prints, DNA profiling, iris imaging, etc[2]. Among all biometric recognition process, fingerprint system is a good way to identify any individual. The paper [4] give information, most researchers use the local ridge orientation of fingerprints for classification. The paper [5] provides FBI information using the classification based on "Henry's Classification" divided into eight parts, the classes are: Plain Arch, Tented Arch, Right Loop, Left Loop, Plain Whorl, Central Pocket Loop, Double Loop, and Accidental Whorl. In present time it is important to identify any one very correctly. According to paper [7], in any big country growing population is part of it, that's why the government also use the fingerprint system to identification. The government use this technique in so many fields that's create citizenship authorization, create passport etc. Not only that IDENT (INS's Automated Biometric Identification System), used to monitor illegal border crossing activity [7], to find any criminal that technique will help us very well. So many another fields are also used this technique 
that's are National and international banks, ATM's, electronic locks, phone locks, car locks etc [10]. Assiya Utzhanova [11] shows that, the fingerprint-based scanning devices are most popular on other biometric based scan.

In the chapter 1 we discuss the basic information about fingerprint and their percentage of uses. In next chapter we know about the initial story of fingerprint. The next section provides data on the world's various kinds of fingerprints. The recognition processes are discussed in chapter 4 . Then next section provides information about a lot of implementation of this method and we recommend some enhancement according to our point of perspective in the last section.

\section{Literature Survey}

Mridula, Priyanka paper [1] provides the data in the previous period, fingerprints are used to determine that many crime scenes are printed marks; the variety of prints is visible prints, prints and latent prints. talk about latent print that are not visible to naked eye, to saw this print we need some dust or methods, this is the initial time to use this technique. After passed time this technique will be developed so much, to detect latent prints power method will be come. In this process we sprinkled this powder in the print point and that's easily visible. Powdering technique will start to use since early 1900s. So many power formulas will be created after this method has been developed. Four types of regular, luminescent, metallic and thermoplastic fingerprint power are generally available. The paper [3] gives the information in 1877 Sir William Harschel in India began to utilize fingerprints on contracts and deeds and registering prisoners, later fingerprint classification impression characterization authority was set up in Kolkata. Two Indian AzizulHaque and Hem Chandra Bose are basically built up a unique classification order system mark, after which it is known as Henry characterization system as indicated by their chief name. Studying Siddapur R K paper [3] we find a data that in 1880Dr. Henry Faulds released the first article on fingerprints in the scientific journal 'Nature' evaluating the function ridge skin for individualization, in particular its use as proof. After that Sir Francis Galton continued to work on it and released information on assessment and identification of fingerprints.

\subsection{Classification of Fingerprint}

Biometric identification is a distinctive (unique) method to identify any individual person very correctly. The paper [1] gives an information of a person most wield individual characteristics are situated in own fingerprints. According to "Henry's classification" [1] provides data, we understand fingerprints are basically three kinds that are Arch, Loop and Whorl. These classes are further divided into five subclasses: Plain Arch, Tented Arch, Left Loop, Right Loop and Whorl.

\section{1) Arch}

Several studies in Arent de Jongh [12] show that arch patterns are found in about 5\% of all fingerprints [2,3]. The defining characteristic of arch patterns is that there is no delta present. Arch models usually show a comparatively horizontal ridge flow, with ridges flowing from the left to the correct side of the fingerprint, while the ridges show an upswing in the center of the fingerprint. According to Arent de Jongh paper [12] the kinds of arches are: Plain arch, tented arch, Tannenbaum, Roofed arch, Pseudo loop, Composite arch. 
In the below figure we can see the common structure of all types of arches -

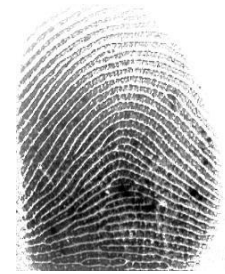

Plain arch

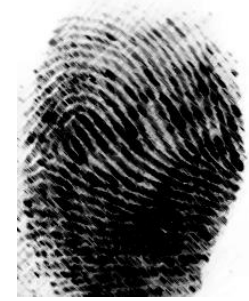

Right roofed arch

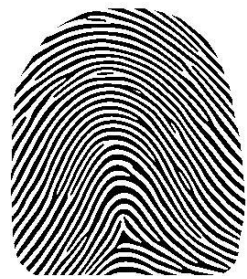

Tented arch

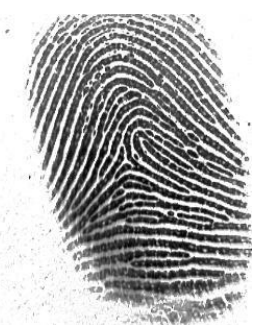

Left pseudo loop

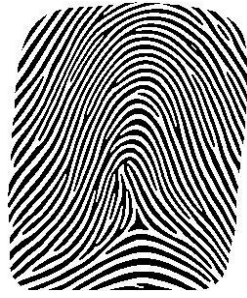

Tannenbaum

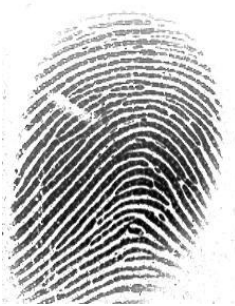

Right pseudo loop

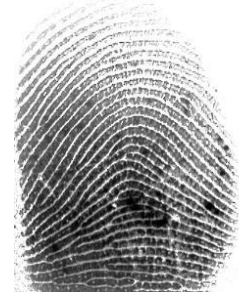

Left roofed arch

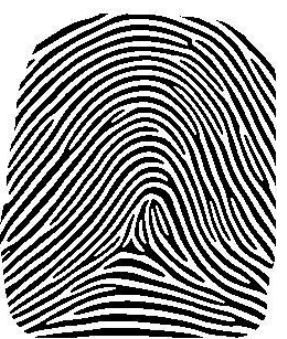

Composite arch

\section{2) Loop}

The paper [1] provides data that one or more of the ridges enter on either side of the fingerprint, re-curve and end in the direction from which the ridges entered. There is one delta and one nucleus in each loop structure. The various structures of loop pattern that's are Plain loop, Pinched loop, Central pocket loop, Inverted loop [12].

In the below figure we shown all loops structure-

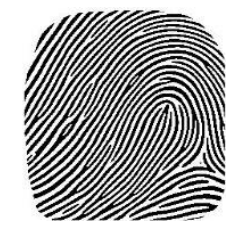

Left plain loop

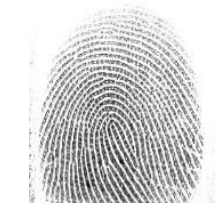

Right plain loop

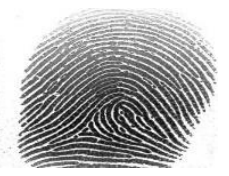

Left central pocket loop Right central pocket loop Left inverted loop $\quad$ Right inverted loop

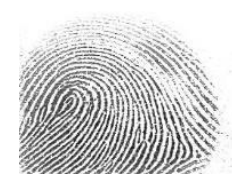

Left pinched loop

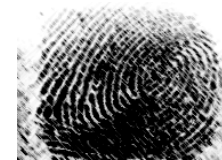

Left central pocket loop Right central pocket loop Left inverted loop Right inverted loop

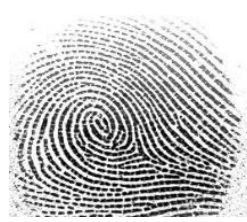

Right pinched loop

We also know in paper [1] the loops are two types one is ulnar loop and another one is Radial loop.

- Ulnar loop -The loop is called the ulnar loop opening towards the small finger. Ulnar loops are named after a bone in the forearm, the ulna bone.

- Radial loop - The loop that opens to the thumb is called the radial loop. Radial loops are named after radius in which the lateral bone is the radius, a bone in the forearm. 
In figure 2.1(a) \& figure 2.1(b) shown the common structure of Ulnar loop \& Radial Loop-

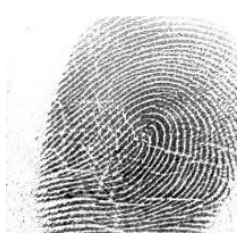

(a)

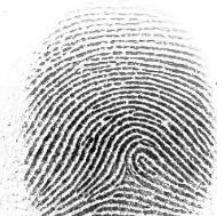

(b)

\section{3) Whorls}

Figure 2.1: (a) ulnar loop (b) Radial loop

A whorl is approximately circular, one or more free recurring ridge lines running all the way around and it's more complicated. It always has at least two deltas points and must have type lines [1]. In paper [1] there are four main kinds of whorls: plain or simple whorl, central pocket whorl, double loop whorl and accidental whorl.

- Plain whorls- In any case, plain whorls must have one ridge making a complete circuit, and an imaginary line from one delta to the next must touch a whorl ridge [1] as shown in fig.3.1.

- Central pocket whorls- At any rate, central pocket whorls must have one ridge making a total circuit and an imaginary line from one delta to the next that can-not touch a ridge of whorls [1] as shown in fig.3.2

- Double loop whorls- Double loop whorls consist of two distinct and unmistakable circles with two distinct and specific shoulders for each core, two deltas and at least one ridges making a complete circuit. In this, at any pace when an imaginary line, a re-bending ridge within the pattern region is sliced or touched [1] (fig. 3.3).

- Some other kind is called accidentals whorl, not in three classifications as shown in fig.3.4.

In fig.3.1, fig.3.2, fig.3.3, fig3.4 we shown the all types -

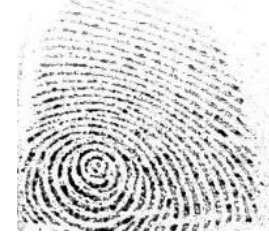

Figure 3.1: plain whorl

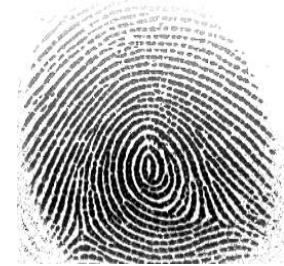

Figure 3.3: central pocket whorl

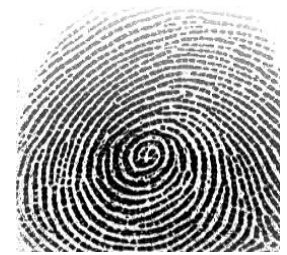

Figure 3.2: central pocket

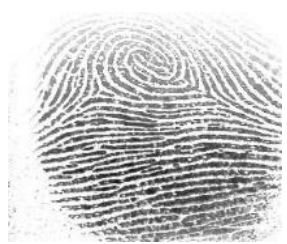

Figure3.4: double loop whorl 
MICHAEL M. S. CHONG [5] gives other whorls images are
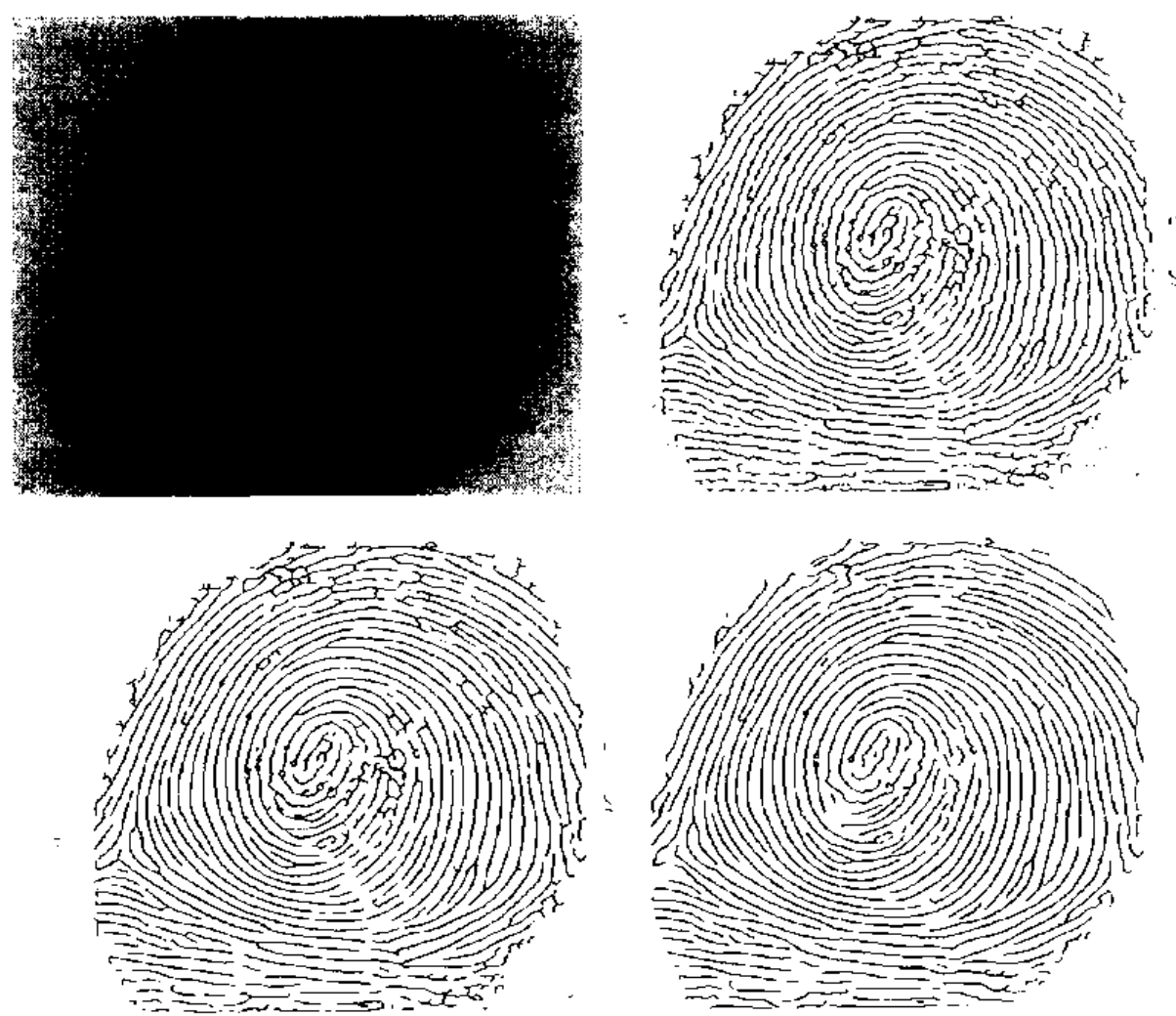

Fig. II. (a) A whorl fingerprint image. (t) Proprowessed whorl fingerprink image. a c Geometric contour

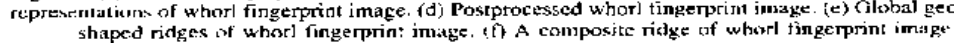

\section{Materials and Methods}

Biometrics recognition is a popular technology for personal identification and protects your personal data; one of them is the fingerprint. In the collection method every information about fingerprint we have to know that how the fingerprint works or what method is using behind the fingerprint. There are several process used to recognized the fingerprints such as Gabor filterbased [4], Minutiae based method [6], Image comparison method [14], Texture based method, Correlation based [14], etc.

Gabor filter-based method is mainly focused on core-point detection rather than other preprocessing steps like smoothing, thinning [4] etc. This proposed method plays main role to performing local ridge orientation, core point detection, and feature extraction. We get information from paper [4] that, this method directly extracted from grey-level fingerprint image, then classify and recognized.

Fingerprint pictures are divided into three main classifications: whorl, loop, arch. Every person's lifetime finger ridge patterns are always same [12]. Usually a fingerprint appears as a sequence of dark rows representing the high, peak portion of the skin of the friction ridge and the valleys between these ridges look like white space and are tiny, shallow portions of the palm skin of the hands [13]. 

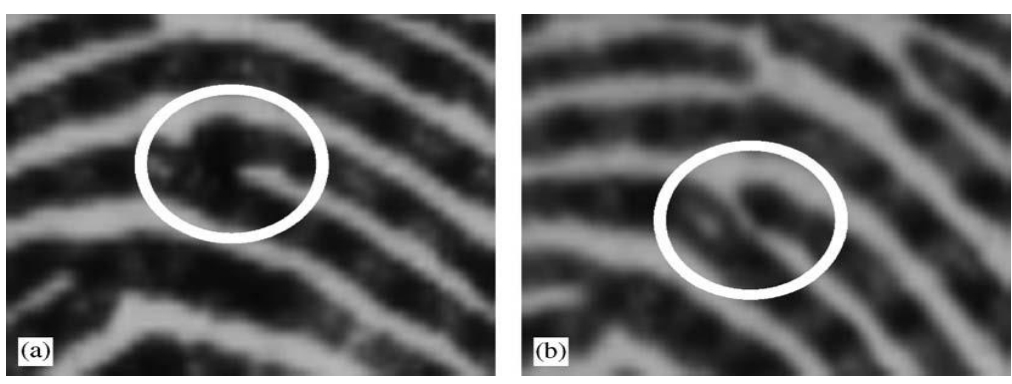

Figure 1: the same minutiae extracted from two different impressions.

In (a) It looks like a bifurcation but in (b) as a ridge ending. Paper [6]

According to A.J. Willis*, L. Myers, Almas M. N. Siddiqui, Rupali Telgad ,Savita Lothe $[9,13]$ Researchers we know about the several steps of proposed minutiae based fingerprint recognition, the following steps are Image acquisition, pre-processing image that involves binarization, an algorithm that creates a 1-bit picture, with 0 as ridges colored with black and 1 as white valleys, Image enhancement which is used to enhance fingerprint quality and overcome some issues use some statistical measures, Feature extraction which using the crossing Number idea for a pixel, and Matching which refers after extraction estimate similarity between two fingerprint, by using neural network.

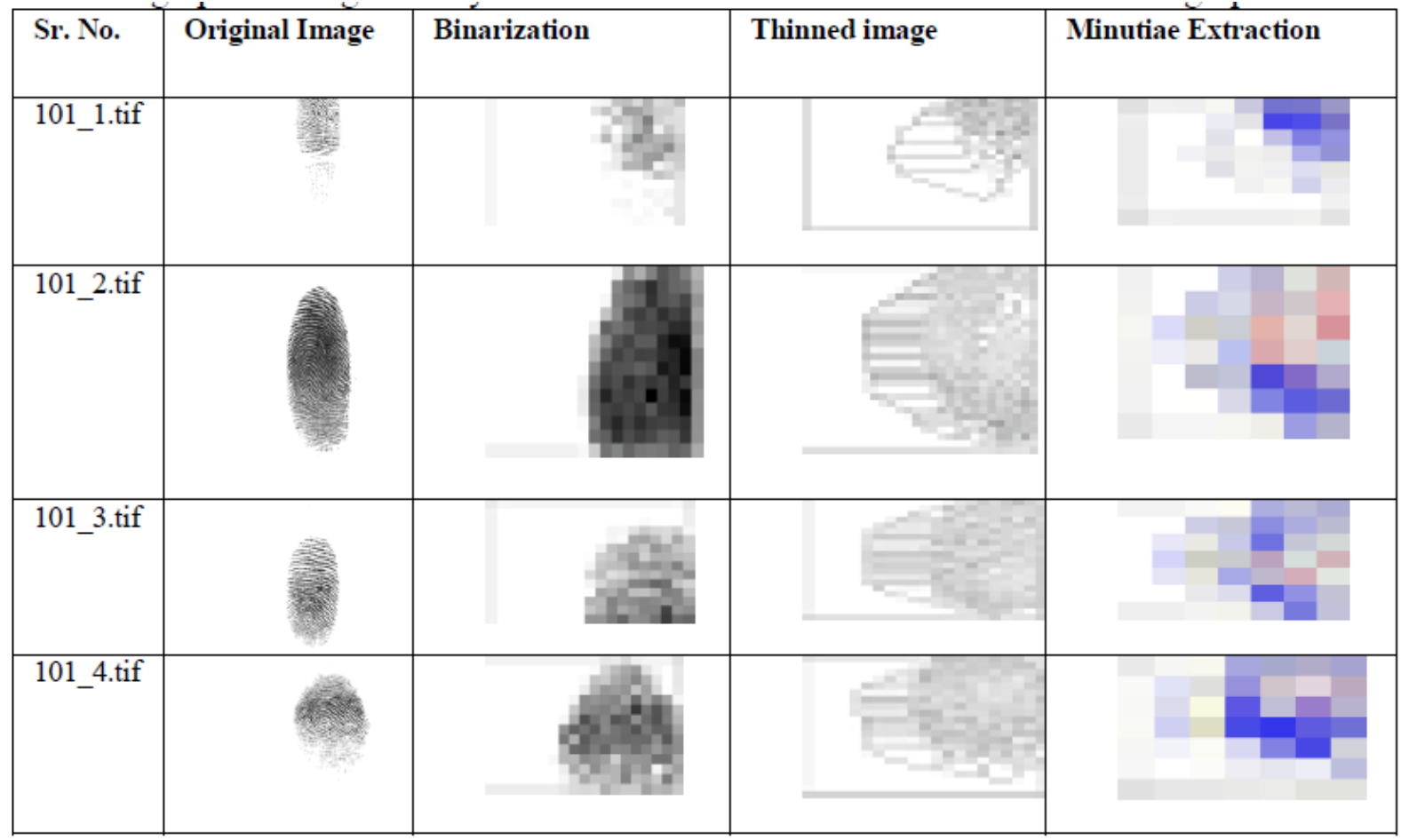

Figure 2: Fingerprint recognition process, paper [13]

Mr. Kannan Subramanian1[14] research gives information that the image based method is one type of correlation method in which compares the sum of multiplication of pixels fingerprint images by using two-dimensional Fast Fourier Transform (FFT) on the template and the input fingerprint however fingerprint is slightly different each capturing time by noise like dirty fingers. 
To give appropriate decision the proposed method using some pre-processing steps like Notation which works on Grey-level images to represent the intensity of the pixel, Normalization based on a pixel operation reducing differences in grey-level values along valleys and ridges, Binarization and Thinning which includes converting the grey-scale image to binary image and setting the ridges of fingerprint of one pixel.

Being interested in Biometric system makes the technology advanced in safety measures, more secure and confidential of their environment. Fingerprint method is one of most common technology that can be captured either with a live-scan fingerprint scanner or with data about ridges and valleys [8]. Fingerprint can be collected by optical sensors. Optical Fibre Sensor is a technique which prefers a fibre-optic plate rather than lens. By reflecting light from valleys and ridges, it defines the fingerprint [8].

We can collect different type of fingerprint by The ID 500 10-Print Live Scan system like flat fingerprint, rolled fingerprint and slap fingerprint [7].

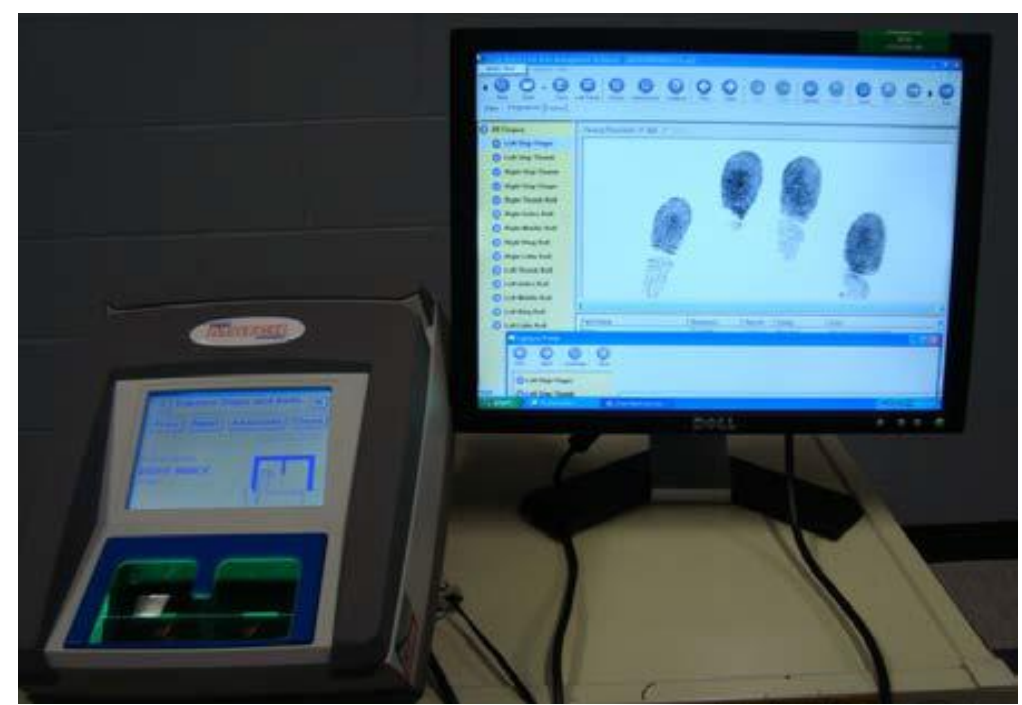

Figure 3: The ID 500 10-Print Live Scan system, paper [7]

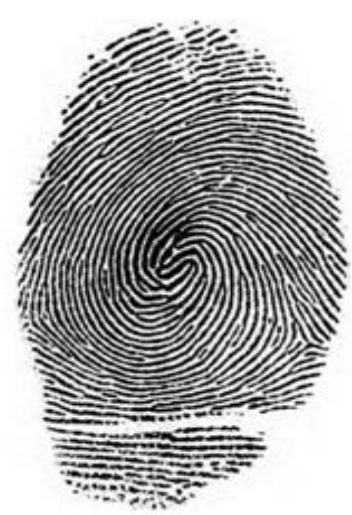

(a)

flat left thumb (African American, male)

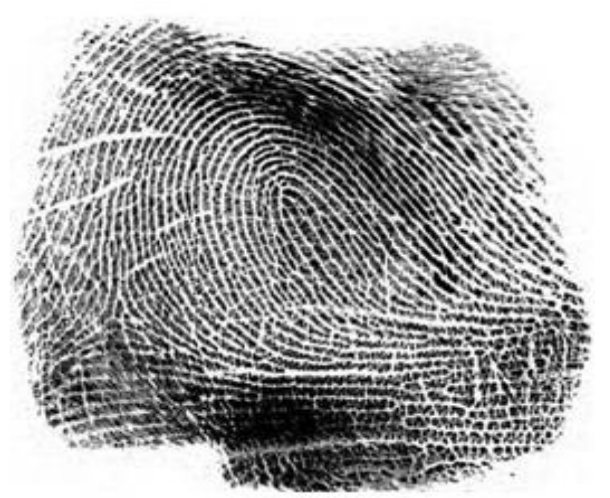

(b)

rolled right thumb

(Asian, male)

Figure 4: Flat fingerprint and rolled fingerprint. Paper [7] 


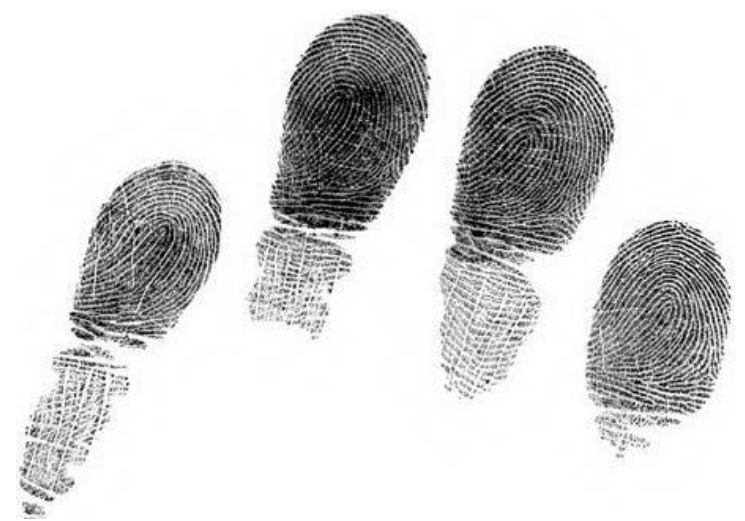

Figure 5: Slap left fingers (Asian, male), paper [7]

A person's fingerprint is collected on any surface which able to use for creating the pattern of matching method. You can capture a fingerprint either full or partial, which is considering as a subset of full fingerprint. For identifying that type of fingerprint there are some kind of process are present like ROI - Region of Interest, a method of segmentation. This process is mainly focused on reference point or core point detection. That's process works on a way which crop a small region reference point. Identifying the fingerprint needs picture resolution. Another process of identifying the fingerprint is Orientation Estimation, which is an action of orienting image to point of a compass. Gradient Based approach is the best method of orientation of ridge [15].

There are also a scheme called Hand geometry that is used for certain hand measurements such as finger width and length. This type of system mainly follows the optical principle. This system is suitable for one-to-one use such as Access control, Time or attendance applications, however where a big proportion of user have access to less disciplined hand geometry in their attitude to the system [16].

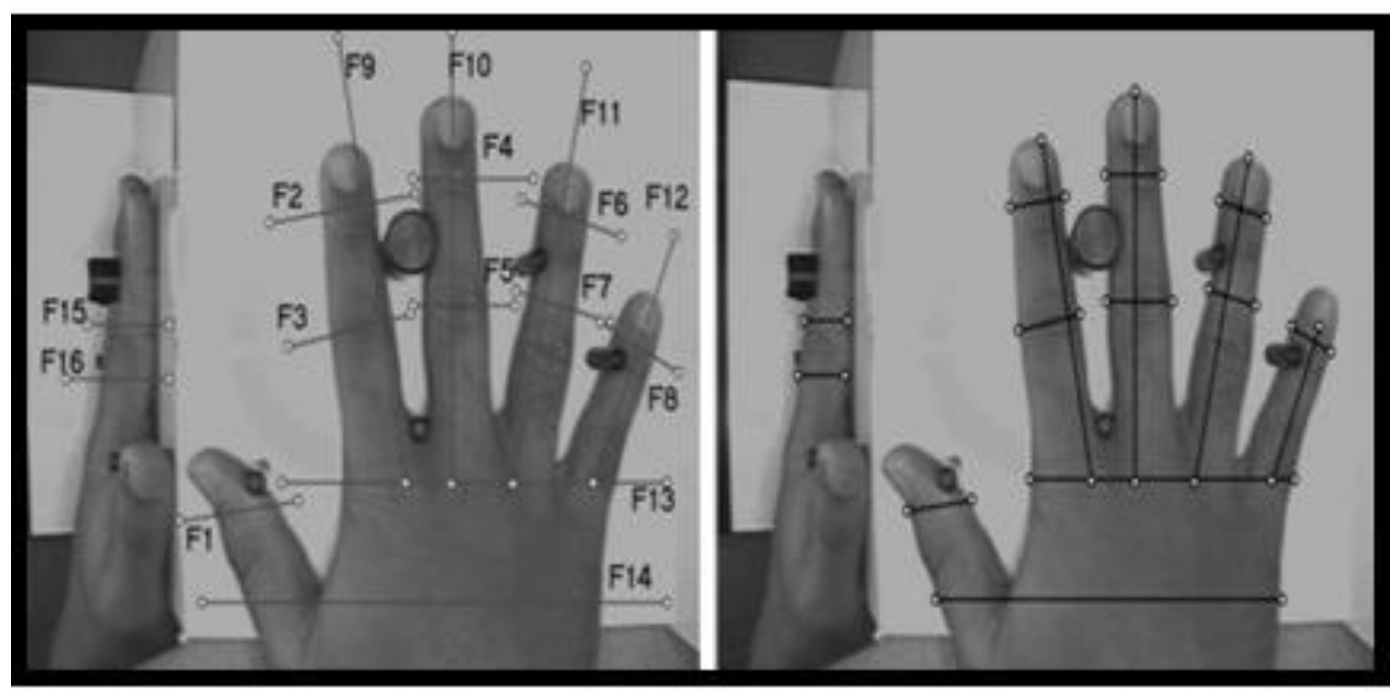

Figure 6: Hand Geometry, paper [17]

Ultimately, we can conclude that biometric fingerprinting primarily follows the two basic values of immutability referring to ridge patterns and uniqueness meaning separate ridge patterns [17]. 


\section{Results and Discussions}

Fingerprints are different for any individual, so that's why this time so many fields are using this method to recognize any individual very correctly. Other biometric characteristic are changeable such as biometric signature, voice commend, face recognition etc. Moreover, the contemporary identity of life today is most essential to safeguard certain records or other stuff, that time fingerprint technology are developed very well, this technology recognition process is very accurate. The paper [11] gives information's government database in 2016 Asia and Africa are used fingerprint technique to give any person's his citizenship. A person provides his fingerprint that's means he gives his own unique identification, so it is very secure process. It is a state-of-theart locking system that is fast to omnipresent and also offers improved safety. To cross any country borders their also used fingerprints to identify any individual bio data. So many other areas also used this method to secure his database, that's banking system, ATMs as PIN, lock any devices like phone and laptop, lock any car etc. Passing days, the world is transformed into digital life, so that very big numbers of individuals use the internet transaction system that we use to safeguard the online transaction process. Japan is planned to test the fingerprint scan to online payment system in 2016[11]. So many researches are proofed that is method is very secure than other biometric recognition methods, that's why in day by day this technique is used another big or small country. In India also government publish a online banking system their also use fingerprint recognize system to secure the banking process.

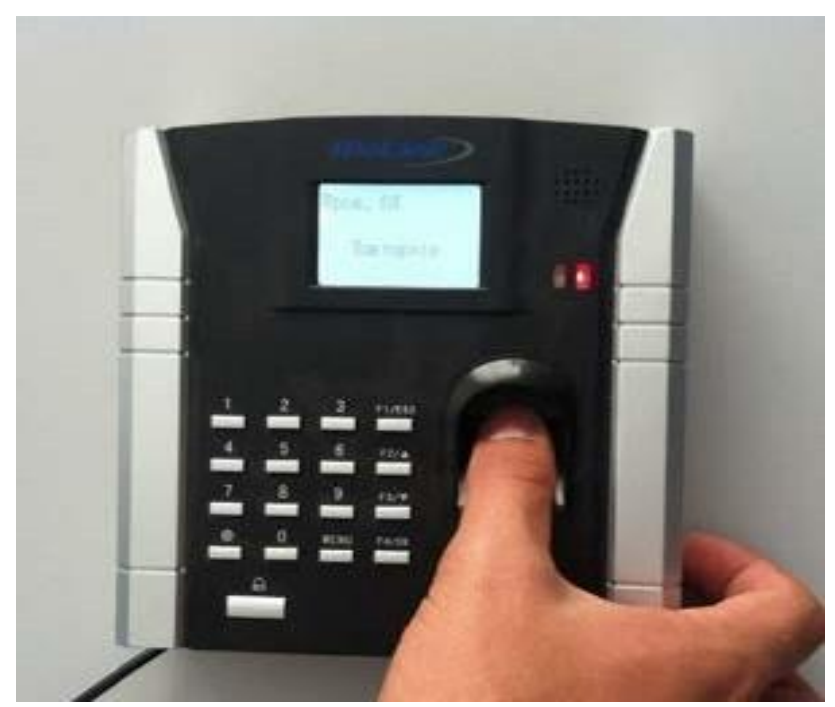

The paper [11] gives in fig.1 biometric attendance machine

\section{Conclusions and Recommendations}

Fingerprint system is one of popular concept to biometric characteristic recognize process to any individual. It is a state-of-the-art locking system that is quick to pervasive and also provides better safety. Chandana, Surendra Yadav, Manish Mathuria [15] research gives information that so many different places are used this technique, but in near future if we use this technique that provide better security in our life. If we use this technique to GOVT Election to identify all peoples individually then we prevent fake person votes, also prevent double vote in one person [15]. Passing days the all over world is converted into digital life so very large amount of people are use 
online transaction system we use this technique to secure the online transaction process. That time, so many techniques are used to scan fingerprint, such as capacitive, image, ultrasonic wave etc. which are safe but are not adequate by requiring more safety objective. In future, identify any unmatched fingerprint then a siren alarm to prevent any kind of thread [10]. For system demanding more security purpose we put multiple fingerprints in a one-time to secure that place such as big museum, bank vault etc [10]. We found information T. Sabhanayagam, Dr. V. Prasanna Venkatesan, Dr. K. Senthamaraikannan paper[17] that other biometric characteristics are not performed very well in real time recognition process, some time you are so nervous that time may be your voice recognition process will be failed but in case of fingerprint recognize system that possibility is gone. To see all scopes of fingerprint system we would say this technique is most useful in future, in fasting growing life this technique is need more security purpose more recognize factors and fast recognize technique.

\section{References}

[1] Mridula, Priyanka Electronics and communication EnggDeptt, Deen bandhu Chhotu Ram University of Science and Technology, Murthal, India, "A Review on Classification of Fingerprint Images”, Volume 9, Issue 3, Ver. III (May - Jun. 2014), PP 61-66.

[2] Bandameedi Lakshmi Narayana1, YerukalaKomeraChinna Rangaiah2, Mohammed Abdul Khalid3," Study of Fingerprint Patterns in Relation to Gender and Blood Group", volume 5, issue 14.

[3] Siddapur R K, "Study on the Relationship between Fingerprint Pattern and Intellectual Performance", International Journal of Medical Toxicology and Forensic Medicine. 2017;7(1): 2631

[4] Chih-Jen Lee and Sheng-De Wang Dept. of Electrical Engineering EE Building, Rm. 441 National Taiwan University Taipei 106, Taiwan. "A Gabor Filter-Based Approach to Fingerprint Recognition".

[5] Michael M. S. Chong, Tan Han Ngee, Liu JunAnd Robert K. L. Gay, "Geometric Framework For Fingerprint Image Classification”, Pattern Recognition, Vol. 30, No. 9, pp. 1475-1488, 1997.

[6] Tsai-Yang Jea*, VenuGovindaraju, "Aminutia-based partial fingerprint recognition system", Center for Unified Biometrics and Sensors, University at Buffalo, State University of New York, Amherst, NY 14228, USA.

[7] Lidong Wang1, *, Cheryl Ann Alexander2, "Fingerprint Patterns and the Analysis of Gender Differences in the Patterns Based on the U Test", International Transaction of Electrical and Computer Engineers System, 2014, Vol. 2, No. 3, 88-92.

[8] Shahzad Memon, Mojtaba Sepasian, Wamadeva Balachandran, "Review of Finger Print Sensing Technologies".

[9] A.J. Willis*, L. Myers, "A cost-e!ective "ngerprint recognition system for use with low-quality prints and damaged "ngertips".

[10] AjinkyaKawale, "Fingerprint based locking system", International Journal of Scientific \& Engineering Research, Volume 4, Issue 5, May-2013.

[11] Assiya Utzhanova1, "Fingerprint Technology and Sustainable Development", European Journal of Sustainable Development (2016), 5, 4, 325-334.

[12] Arent de Jongh, 1 Ph.D.; Anko R. Lubach, 1; Sheryl L. Lie Kwie, 1 M.A.; and Ivo Alberink, 1 Ph.D.," Measuring the Rarity of Fingerprint Patterns in the Dutch Population Using an Extended Classification Set", J Forensic Sci, January 2019, Vol. 64, No. 1.

[13] Almas M. N. Siddiqui1, Rupali Telgad2, Savita Lothe3, Dr. P. D. Deshmukh1, "Fingerprint Recognition System for Person Identification Using Termination and Bifurcation Minutiae", IOSR Journal of Computer Engineering (IOSR-JCE) e-ISSN: 2278-0661, p-ISSN: 2278-8727, PP 33-38 
[14] Mr. Kannan Subramanian1, “Image Based Fingerprint Verification”, Vol. 2, Issue 5, May 2014.

[15] Chandana, Surendra Yadav, PhD, Manish Mathuria, "Fingerprint Recognition based on Minutiae Information", International Journal of Computer Applications (0975 - 8887), Volume 120 - No.10, June 2015

[16] Shilpa Shrivastava1, "Biometric: Types and its Applications".

[17] T. Sabhanayagam1, Dr. V. Prasanna Venkatesan2 and Dr. K. Senthamaraikannan3, "A Comprehensive Survey on Various Biometric Systems", International Journal of Applied Engineering Research ISSN 0973-4562 Volume 13, Number 5 (2018) pp. 2276-2297.

[18] Kaoru UCHIDA, "Fingerprint identification", NEC journal of advance technology, volume 2, no 1.

[19] JinweiGu, "Fingerprint Recognition by Combining Global Structure and Local Cues", IEEE transactions on image processing, vol. 15, no.7, july 2006.

[20] T. Amornraksa and S. Tachaphetpiboon, "Fingerprint recognition using DCT features", Institute of Engineering \& Technology Volume 42, Issue 9.

[21] Pierre Baldi, Yves Chauvin, "Neural Networks for Fingerprint Recognition", Neural Computation - May 1993

[22] Louis coetzee and elizabeth c. botha, "fingerprint recognition in low quality images ", Pattern Recognition, Vol. 26, No. 10, pp. 1441 1460, 1993.

[23] Faisal Farooq, Ruud M. Bolle, Tsai-Yang Jea and NaliniRatha, "Anonymous and Revocable Fingerprint Recognition ", IEEE Conference on Computer Vision and Pattern Recognition, 17-22 June 2007.

[24] Marius Tico, EeroImmonen, Pauli Ramo, Pauli Kuosmanen, and Jukka Saarinen, "fingerprint recognition using wavelet features, The 2001 IEEE International Symposium on, Volume: 2.

[25] andrew k. hrechak and james a. mchugh," automated fingerprint recognition using structural matching", Pattern Recognition, Vol .23, No .8, pp, 893 904, 1990.

[26] Koichi Ito†, Ayumi Morita $\uparrow$, Takafumi Aoki†, Tatsuo Higuchi†, Hiroshi Nakajima\$, and Koji Kobayashi\$, "A Fingerprint Recognition Algorithm Using Phase-Based Image Matching for LowQuality Fingerprints, IEEE International Conference on Image Processing 2005

[27] emanuela marasco, arun ross, "A Survey on Antispoofing Schemes for Fingerprint Recognition Systems", ACM Computing Surveys, Vol. 47, No. 2, Article 28.

[28] MunishKumar1, Priyanka2, "Fingerprint Recognition System: Issues and Challenges", ISSN: 2321-9653; IC Value: 45.98; SJ Impact Factor: 6.887 Volume 6 Issue II, February 2018.

[29] Dibyendu Nath1, Saurav Ray2, Sumit Kumar Ghosh3, "Fingerprint Recognition System: Design \& Analysis".

[30] Anil K. Jain, Fellow, IEEE, and JianjiangFeng, Member, IEEE, "Latent Fingerprint Matching".

[31] Lidong Wang1, *, Cheryl Ann Alexander2, "Fingerprint Patterns and the Analysis of Gender Differences in the Patterns Based on the U Test", International Transaction of Electrical and Computer Engineers System, 2014, Vol. 2, No. 3, 88-92.

[32] Asan Baker Kanbar, "Fingerprint Identification for Forensic Crime Scene Investigation ", Asan Baker Kanbar, International Journal of Computer Science and Mobile Computing, Vol.5 Issue.8, August- 2016, pg. 60-65.

\footnotetext{
*Corresponding author.

E-mail address: prianka@ ecmt.in
} 